\title{
Size Effects in Transport Properties of PbSe Thin Films
}

\author{
E.I. ROGACHEVA (i), ${ }^{1,2,3}$ O.N. NASHCHEKINA, ${ }^{1}$ and S.I. MENSHIKOVA ${ }^{1}$ \\ 1.-National Technical University "Kharkov Polytechnic Institute", 21 Frunze St., Kharkov \\ 61002, Ukraine. 2.—e-mail: rogacheva@kpi.kharkov.ua. 3.—e-mail: rogachova.olena@gmail.com
}

\begin{abstract}
This paper presents an overview and analysis of our earlier obtained experimental results on the dependences of kinetic properties of single PbSe quantum wells and PbSe-based superlattices on the PbSe layer thickness $d$. The observed oscillatory character of these dependences is attributed to quantum size effects due to electron or hole confinement in quantum wells. Some general regularities and factors that determine the character of these quantum size effects are established. The influence of the oxidation processes and doping on the $d$-dependences of the transport properties is revealed. A periodic change in the conductivity type related to quantum size oscillations is detected. It is shown that the experimentally determined values of the oscillation period $\Delta d$ are in good agreement with the results of theoretical calculations based on the model of a rectangular quantum well with infinitely high walls, taking into account the dependence of the Fermi energy $\varepsilon_{\mathrm{F}}$ on $d$ and the availability of subbands below $\varepsilon_{F}$. It is established that the $\Delta d$ value for the superlattices is practically equal to the $\Delta d$ value observed for the single $\mathrm{PbSe}$ thin film.
\end{abstract}

Key words: PbSe, quantum well, width, superlattice, thermoelectric properties, galvanomagnetic properties, quantum size effects

\section{INTRODUCTION}

If the size of a system, in at least one dimension, becomes comparable to the de Broglie wavelength $\lambda_{\mathrm{D}}$, quantum size effects (QSEs), which manifest themselves in the dependences of physical properties on the system size, are observed. ${ }^{1,2}$ Electron confinement in low-dimensional (LD) structures opens new ways for controlling properties of solids, stimulating theoretical and experimental studies of specific properties of systems whose behavior can be described only in terms of quantum mechanics. Such studies are additionally stimulated by recent technological advances in preparation of high-quality nanostructures and designing devices based on QSEs.

Two-dimensional (2D) systems include thin films, quantum wells (QWs), and multiple-QW structures such as superlattices (SLs). In thin film with thickness $d$, the movement of electrons is confined

(Received November 8, 2016; accepted March 25, 2017; published online April 26, 2017) in the $z$-direction perpendicular to the film surface, while electrons can move freely in the $x$ - and $y$ directions; i.e., for electrons, a film represents a QW of width $d$ and depth equal to the work function. Using the simplest model of a rectangular well with infinitely high walls ${ }^{1,2}$ and assuming a parabolic dispersion law for particles and specular reflection from $\mathrm{QW}$ walls, one can write the energy quantization rule as

$$
E_{n}=\frac{\pi^{2} \hbar^{2} n^{2}}{2 m_{z}^{*} d^{2}}+\frac{\hbar^{2} k_{x}^{2}}{2 m_{x}^{*}}+\frac{\hbar^{2} k_{y}^{2}}{2 m_{y}^{*}},
$$

where $E_{n}$ are the discrete energy levels in the $\mathrm{QW}$, $m_{x}^{*}, m_{y}^{*}, k_{x}$, and $k_{y}$ are the components of the effective mass and electron wavevector, respectively, for motion parallel to the film surface, $m_{z}^{*}$ is the effective mass for motion perpendicular to the film surface, and $n$ is the quantum number. Space quantization leads to splitting of an energy band into $2 \mathrm{D}$ subbands. As a result, the electron energy spectrum is a superposition of a continuous spectrum with a parabolic dispersion law corresponding 
to the movement in the $x-y$ plane, and the discrete spectrum arising from the confined movement along the $z$ axis. For given $n$, the energy passes through a continuous series of values, called a subband, and the energy spectrum of the thin film consists of overlapping subbands.

QSEs can be observed in thin films when a number of conditions are fulfilled, in particular electron gas degeneracy, sufficiently high charge carrier mobilities, and high-quality surfaces. It is possible to detect size quantization of the energy spectrum by studying transport phenomena. In Ref. 3, it was theoretically predicted that, as a result of size quantization of the energy spectrum in $2 \mathrm{D}$ structures, the dependences of thermodynamic and kinetic properties on $d$ must exhibit oscillatory behavior with period $\Delta d$ :

$$
\Delta d=\frac{\lambda_{\mathrm{F}}}{2}=\frac{h}{\sqrt{8 m_{z}^{*} \varepsilon_{\mathrm{F}}}},
$$

where $\varepsilon_{\mathrm{F}}$ is the Fermi wavelength. Of course, such a model can only be used for rough estimates, which do not take into account that the energy bands in $\mathrm{PbSe}$ are anisotropic, with ellipsoidal constantenergy surfaces having two effective mass components (longitudinal and transverse) and nonparabolic dependence of the charge carrier energy on quasimomentum at sufficiently high energy. ${ }^{4}$ This model does not take into consideration the nonidentity of the barriers at the interfaces or the presence of defects.

For very small thicknesses, the bottom of the first lateral subband lies above the Fermi level $\varepsilon_{F}$ and electrical conductivity is absent. With increasing $d$, the first subband moves down, and when its bottom intersects $\varepsilon_{\mathrm{F}}$, the film becomes conducting. The critical thickness $d_{1}$, at which the first subband passes through $\varepsilon_{\mathrm{F}}\left(E_{1}=\varepsilon_{\mathrm{F}}\right)$, can be estimated as $d_{1}=h / \sqrt{ } 8 m_{z} \varepsilon_{\mathrm{F}}=\Delta d=\lambda_{\mathrm{F}} / 2$. It therefore follows that $\Delta d$ can be determined from the position of the first extremum. If $d$ is further increased by $\lambda_{\mathrm{F}} / 2$, the next subband drops below $\varepsilon_{\mathrm{F}}$ and starts to contribute to conductivity. The number of populated subbands $N$ changes in a step-like fashion. At thicknesses for which a new subband starts to be populated and a new wavefunction starts to contribute to the electron concentration, the density of states changes in a jump-like fashion, which determines the oscillatory behavior of the density of states as a function of $d$. Oscillations in the density of states must lead to oscillations in the charge carrier mobility, electrical conductivity, and Seebeck coefficient. If we assume that a QW represents an ensemble of electrons with fixed chemical potential, corresponding to $\varepsilon_{\mathrm{F}}$ in thick films, then with changing $d$, the electron concentration in the QW must oscillate relative to the constant value of $\varepsilon_{\mathrm{F}}$. Within a single subband (in the range of thicknesses between $d_{n}$ and $d_{n+1}$ ), the electron concentration first increases, reaches a maximum, then decreases.
Thus, the Fermi level calculated from the bottom of the conduction band in a bulk sample does not change position with increasing $d$, while electron subbands slide down past it and gradual filling of lateral subbands takes place.

It should also be noted that, when higher subbands are occupied, it is necessary to consider the contribution to the transport properties from different subbands and intersubband scattering processes, which can result in additional broadening of levels.

In metallic films, $\lambda_{\mathrm{F}}$ and $\Delta d$ are comparable to interatomic distances, so to study QSEs using transport property measurements, it is necessary to grow very thin films with high degree of structural perfection. Semimetals and semiconductors, which have significantly larger $\lambda_{F}$ values, represent more convenient systems for studying QSEs by measuring their transport properties. ${ }^{5}$ This is why quantum oscillations in the transport properties of thin films with changing $d$ were observed for the first time for semimetallic Bi films, ${ }^{6}$ and soon after that for semiconducting compounds $\mathrm{InSb}$ and $\mathrm{Cd}_{3} \mathrm{As}_{2}{ }^{7,8}$ However, the number of studies related to quantum size oscillations in semiconducting $\mathrm{QWs}$ still remains very limited.

\section{STUDY AIMS}

$\mathrm{PbSe}$ semiconductor compound belongs to the IVVI compounds, which are well known as promising materials for use in thermoelectric (TE), optoelectronic, and other applications. ${ }^{4,9}$ Recently, interest in these compounds has been further stimulated due to theoretical predictions and subsequent experimental evidence of a significant enhancement in the TE figure of merit $Z T\left(Z=S^{2} \sigma T / \lambda\right.$, where $S$ is the Seebeck coefficient, $\sigma$ is the electrical conductivity, $\lambda$ is the thermal conductivity, and $T$ is the absolute temperature) in LD structures, in particular in IV-VI-based SLs, compared with their bulk counterparts. ${ }^{10,11}$ Therefore, in the physics of thermoelectricity and TE materials, there is currently a strong focus on studying LD systems, which is related to their great potential for TE applications. A sufficiently small effective mass in the direction perpendicular to the direction of growth and high charge carrier mobility make IV-VI compounds convenient materials for studying QSEs using measurements of transport properties.

During the last 15 years approximately, we have carried out a cycle of experimental studies ${ }^{12-22}$ of the room-temperature dependences of $\sigma, S$, the Hall coefficient $R_{\mathrm{H}}$, and Hall charge carrier mobility $\mu_{\mathrm{H}}$ on the PbSe layer thickness (QW width) in PbSebased QWs and SLs. The initial materials used for growing single $\mathrm{PbSe}$ layers were lead selenide crystals with different charge carrier types and concentrations: (1) stoichiometric $p$-PbSe crystals with $p=3 \times 10^{18} \mathrm{~cm}^{-3},{ }^{14}$ (2) nonstoichiometric $n$-PbSe crystals with $n=3 \times 10^{18} \mathrm{~cm}^{-3},{ }^{15-17}$ and 
(3) $n$-PbSe crystals heavily doped with $\mathrm{PbCl}_{2}$ $\left(n=3 \times 10^{20} \mathrm{~cm}^{-3}\right) \cdot{ }^{18-20}$ We also studied (001)KCl/ $n$-PbSe/EuS SLs with various thicknesses of the PbSe layer. ${ }^{21,22}$ All QWs and SLs were covered with protective layers, except the films prepared from nonstoichiometric $n$-PbSe crystals, which were studied without protective covering. ${ }^{12,13}$ In all cases, $(001) \mathrm{KCl}$ crystals were used as substrates. PbSe monocrystalline thin films were prepared by thermal evaporation of $\mathrm{PbSe}$ crystals in vacuum $\left(10^{-5} \mathrm{~Pa}\right.$ to $\left.10^{-6} \mathrm{~Pa}\right)$ with subsequent deposition onto $(001) \mathrm{KCl}$ at $520 \pm 10 \mathrm{~K}$. EuS, EuSe or $\mathrm{Al}_{2} \mathrm{O}_{3}$ layers used for protecting PbSe films from oxidation were prepared by electron-beam evaporation. Transmission electron microscopy studies showed that $\mathrm{PbSe}$ films grew epitaxially on $(001) \mathrm{KCl}$ according to the vapor-crystal growth mechanism. Film growth started with formation of islands. At $d \approx 4 \mathrm{~nm}$ to $10 \mathrm{~nm}$, the films showed semicontinuous channel structure, and at $d>10 \mathrm{~nm}$, the films were practically continuous. EuS and EuSe also grew epitaxially on the PbSe surface in (001) orientation. Being wide-gap semiconductors, EuS, EuSe, and $\mathrm{Al}_{2} \mathrm{O}_{3}$ make a negligible contribution to the conductivity. In Ref. 21, we described in detail the preparation technique and growth mechanism for $(001) \mathrm{KCl} / \mathrm{PbSe} / \mathrm{EuS}$ SLs. In Ref. 22, the thickness of PbSe layers $\left(d_{0}\right)$ in SLs was varied in the range of $0.5 \mathrm{~nm}$ to $15 \mathrm{~nm}$, whereas the thickness of EuS layers was $\sim 10 \mathrm{~nm}$. The upper (covering) EuS layer was $30 \mathrm{~nm}$ to $40 \mathrm{~nm}$ thick. The number of SL periods was varied from 5 to 25 . The Hall coefficient $R_{\mathrm{H}}$ and electrical conductivity $\sigma$ were measured using a conventional direct-current (dc) method in magnetic field of $0.9 \mathrm{~T}$ on Hall double cross-shaped samples. Six ohmic contacts were prepared by soldering In to the film surface. The measurement error for $R_{\mathrm{H}}$ and $\sigma$ did not exceed $5 \%$. The Hall charge carrier mobility $\mu_{\mathrm{H}}$ was calculated as $\mu_{\mathrm{H}}=R_{\mathrm{H}} \sigma$, and the charge carrier concentration $n$ was determined as $n=r /\left(R_{\mathrm{H}} e\right)$, assuming the Hall factor $r$ to be 1 . The Seebeck coefficient $S$ was determined with respect to $\mathrm{Cu}$ with an error of less than $3 \%$. All measurements were carried out on samples freshly extracted from the vacuum chamber at $300 \mathrm{~K}$.

The purpose of the present work is to reveal some factors that determine the character of the QSEs in these PbSe-based nanostructures and establish some general regularities on the basis of analysis of our experimental results obtained for a sufficiently extensive range of studied materials. We do not include herein our experimental and theoretical results related to classical size effects in PbSe thin films.

\section{RESULTS AND DISCUSSION}

\section{Influence of Oxidation in Air on TE Properties of PbSe Thin Films}

To realize conditions under which the observation of quantum oscillations in $\mathrm{PbSe} \mathrm{QWs}$ becomes possible, it is necessary to overcome a number of problems connected with preparation of high-quality QWs. One such problem is oxidation of the thin-film surface. This problem is very relevant for IV-VI compounds, because oxygen acts as an acceptor, causing the appearance of $p$-type charge carriers, whose presence can radically change the transport properties of crystals and thin films. However, to the best of the authors' knowledge, data on oxidation have only been reported for relatively thick $(d=0.1 \mu \mathrm{m}$ to $10 \mu \mathrm{m})$ films of IV-VI semiconductors, ${ }^{23-25}$ and there are no corresponding data for nanosized thin films. At the same time, with decreasing $d$, the role of a surface layer, exhibiting $p$-type conductivity, becomes increasingly important in determining the thin-film transport properties. Changing $d$, while keeping other parameters the same, makes it possible to vary the contribution of the oxidized surface layer to the transport coefficients in a controlled way. By carrying out measurements on freshly prepared films at room temperature, one can exclude the role of diffusion processes and consider oxidation as only a surface effect. By conducting studies on epitaxial films, one can exclude any contributions made by oxidation of grain boundaries.

We measured the $d$-dependences of $\sigma, S, R_{\mathrm{H}}$, and $\mu_{\mathrm{H}}$ of PbSe thin films ( $d=4 \mathrm{~nm}$ to $200 \mathrm{~nm}$ ), freshly prepared and exposed to air at room temperature. $^{12,13}$ It was shown (Fig. 1) that oxidation leads to a sharp change in the PbSe film properties, including a change in the sign of the dominant carrier type from $p$ to $n$ at $d \leq 80 \mathrm{~nm}$. Using a twocarrier model for thin films $(d<50 \mathrm{~nm})$ and a twolayer model for thick films $(d>50 \mathrm{~nm})$ allowed us to give a satisfactory qualitative interpretation of the observed experimental $d$-dependences of the transport properties.

It follows from the results obtained in Refs. 12 and 13 that it is impossible to establish the true thickness dependences of the PbSe QW transport properties, and thus reveal the physical effects occurring at nanosize level, without taking special measures to protect the film from oxidation. This is why we studied QSEs in PbSe thin films covered with a protective layer (EuS, EuSe, $\mathrm{Al}_{2} \mathrm{O}_{3}$ ).

\section{Dependence of Conductivity Type of PbSe Thin Films on Initial Crystal Composition and Film Thickness}

As follows from Eq. 2, an important parameter determining the oscillatory character of the $d$ dependences of the transport properties is the charge carrier concentration $n(p)$, which determines the number of populated subbands and the $\Delta d$ value. By varying $n(p)$, one can control the properties of LD structures such as heterostructures, SLs, quantum wires, etc. Analysis of our experimental data allowed us to reveal the effect of $n$ - or $p$-type doping (shift of the Fermi level) on the oscillatory phenomena. 

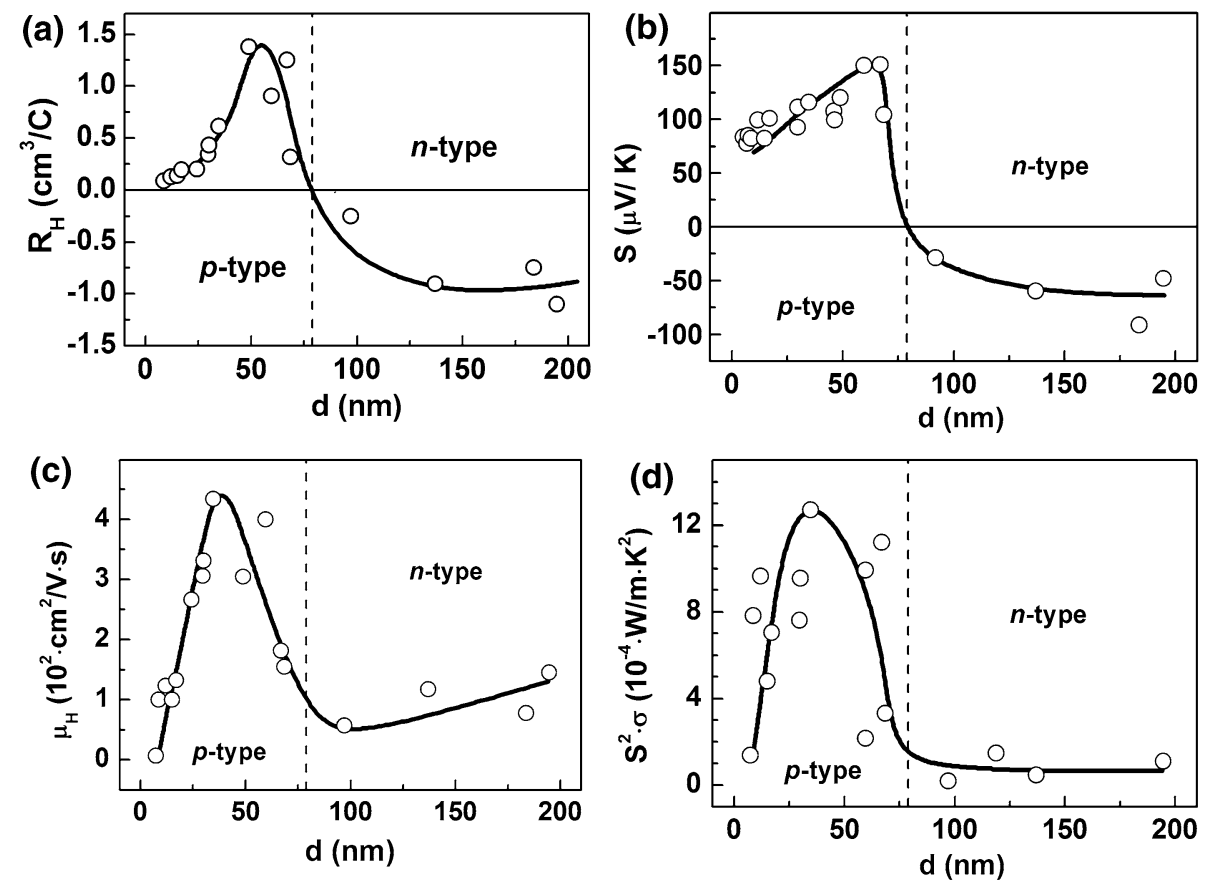

Fig. 1. Room-temperature dependences of Hall coefficient $R_{\mathrm{H}}$ (a), Seebeck coefficient $S$ (b), charge carrier mobility $\mu_{\mathrm{H}}$ (c), and power factor $P=S^{2} \sigma(\mathrm{d})$ on PbSe film thickness $d$. The dashed vertical line denotes the $d$ value at which the dominant carrier sign changes. ${ }^{12,13}$

It was established that, in films prepared using stoichiometric $p$-PbSe crystals as initial material, the conductivity type depended on $d$ : when $d$ increased to $\sim 20 \mathrm{~nm}$, inversion of the dominant carrier sign $(p \rightarrow n)$ occurred, then in the thickness range of $d=20 \mathrm{~nm}$ to $60 \mathrm{~nm}$, the conductivity sign changed in a periodic fashion accompanied by oscillations of the kinetic coefficients, whereas at higher values of $d$, films exhibited $n$-type conductivity (Fig. 2). When $n$-type PbSe crystals were used as initial material for thin-film preparation, the films exhibited $n$-type conductivity throughout the entire thickness range (Figs. 3 and 4).

One can suggest that the appearance of $n$-type conductivity in the films grown from $p$-PbSe crystals is due to partial reevaporation of Se from the heated substrate in the process of condensation. As $\mathrm{PbSe}$ has a two-sided homogeneity region in the $\mathrm{Pb}-\mathrm{Se}$ system and PbSe crystals can be of both $n$ - and $p$-type, even slight deviation from the stoichiometric composition can lead to a change in the conductivity type. On the other hand, the inversion of the conductivity sign under changing $d$ can be caused by a change in the thermodynamic equilibrium conditions in thin films as compared with crystals due to an increase in the contribution of the surface energy to the total energy of the crystal with decreasing $d$. This may lead not only to a change in the equilibrium defect concentration but also to a change in the predominant defect type.

The periodic alternation of the conductivity sign accompanying the $d$-dependent oscillations in the kinetic properties can be attributed to high sensitivity of the electron subsystem to external factors (in this case, to changes in $d$ ) under size quantization at thicknesses close to the inversion point. This assumption is confirmed by the fact that, in the PbSe films obtained from $n$-PbSe crystals and exhibiting $n$-type conductivity throughout the entire thickness range, no changes in the conductivity sign in the process of the oscillations were observed. ${ }^{15-20}$ A similar phenomenon was observed in $p$-PbTe thin films prepared using $p$-PbTe crystals heavily doped with $\mathrm{Na}$ as initial material. Due to high hole concentration in the thin films, no changes in the conductivity sign in the process of the quantum oscillations were registered. ${ }^{26}$

It also follows from the obtained data that, when stoichiometric $p$-PbSe crystals are used as initial material, $p$-type conductivity can be preserved only at sufficiently small thicknesses $d$, even in films protected from oxidation. It can be suggested that PbSe thin films will exhibit $p$-type conductivity in a wider range of thicknesses if the hole concentration in the PbSe crystal used as the initial material for thin-film preparation were higher (i.e., if the $\mathrm{PbSe}$ crystal were doped with an acceptor impurity). It is also shown that the inversion of the conductivity type under changing $d$ makes it possible to study quantum size effects caused by energy spectrum quantization for both electrons and holes using the same specimen.

\section{Quantum Size Effects in PbSe Quantum Wells}

For all PbSe-based QWs, in a certain range of $d$, oscillatory behavior of the dependences of the transport properties on $d$ is observed. The $\Delta d$ value 

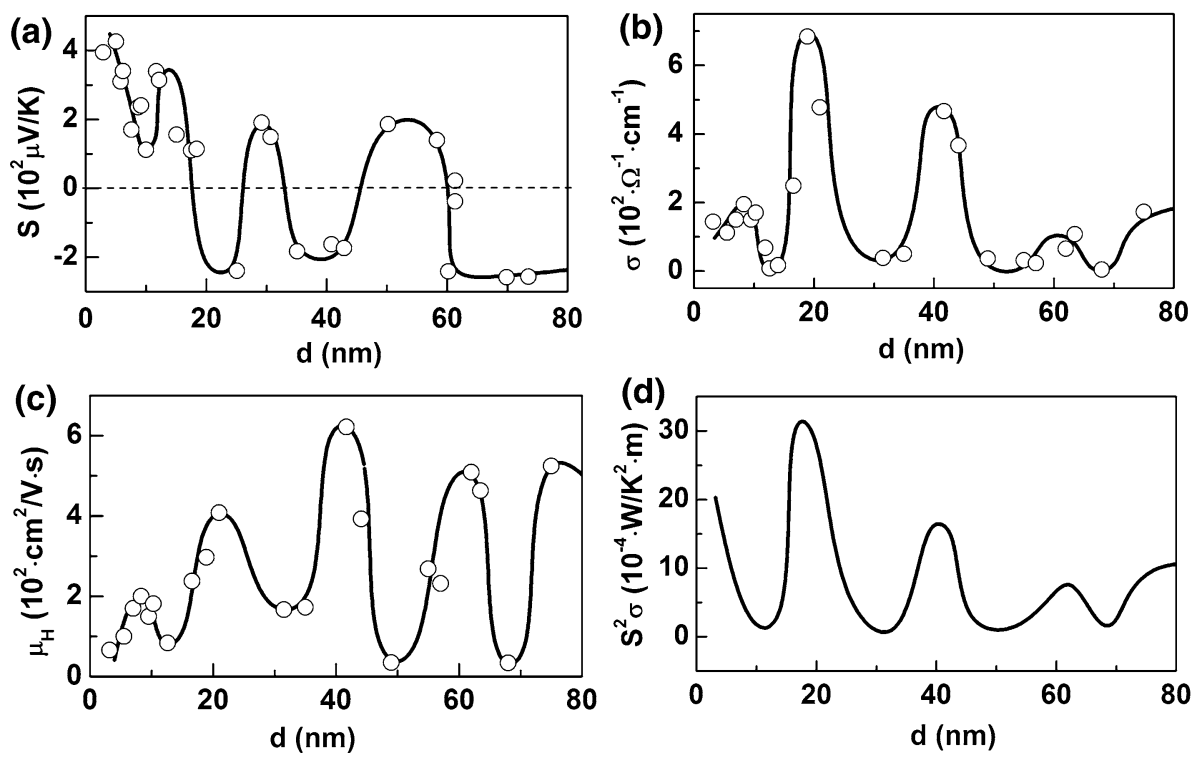

Fig. 2. Seebeck coefficient $S$ (a), electrical conductivity $\sigma$ (b), charge carrier mobility $\mu_{\mathrm{H}}$ (c), and thermoelectric power factor $P=S^{2} \sigma$ (d) as functions of PbSe layer thickness $d$ in $\mathrm{KCl} / \mathrm{PbSe} /$ EuSe structures. ${ }^{14}$

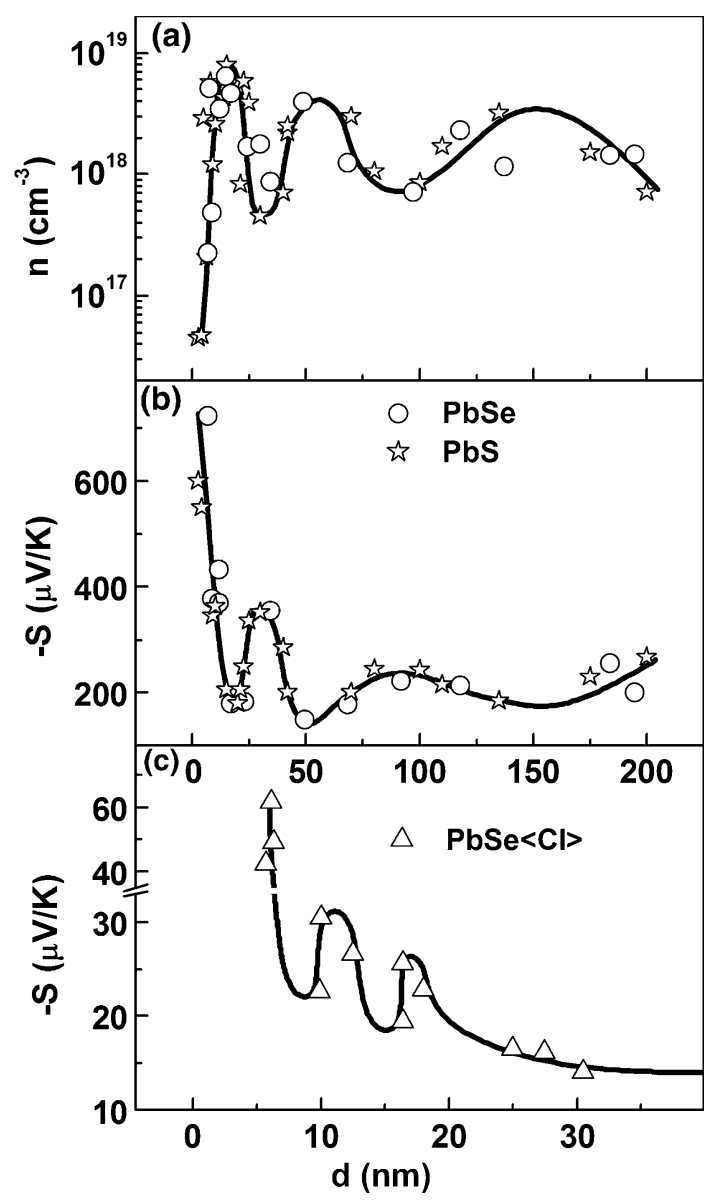

Fig. 3. Room-temperature dependences of charge carrier concentration $n$ (a) and Seebeck coefficient $S$ (b, c) on PbS (a, b), PbSe (a, b), and $\mathrm{PbSe}\langle\mathrm{Cl}\rangle$ (c) layer thickness $d$ in $\mathrm{KCl} / \mathrm{PbS} / \mathrm{EuS},(001) \mathrm{KCl} /$ $\mathrm{PbSe} / \mathrm{EuS}$, and $\mathrm{KCl} / \mathrm{PbSe}\langle\mathrm{Cl}\rangle / \mathrm{Al}_{2} \mathrm{O}_{3}$ quantum wells. ${ }^{15-18}$ was determined experimentally using the value of $d$ corresponding to the position of the first extremum in the dependences of all kinetic coefficients as well as using the mean distance between the minima and maxima. It is natural to suggest that the oscillatory behavior of the $d$-dependences of the transport properties results from the size quantization of the energy spectrum occurring in the $2 \mathrm{D}(001) \mathrm{KCl} /$ $\mathrm{PbSe} / \mathrm{EuS}\left(\mathrm{EuSe}, \mathrm{Al}_{2} \mathrm{O}_{3}\right)$ structures. The PbSe layer is placed between two insulators-the substrate (KCl) and the protective layer (EuS, EuSe or $\mathrm{Al}_{2} \mathrm{O}_{3}$ ) - which confine the motion of charge carriers in the direction perpendicular to the thin-film surface. This is why the $(001) \mathrm{KCl} / \mathrm{PbSe} / \mathrm{EuS}(\mathrm{EuSe}$, $\mathrm{Al}_{2} \mathrm{O}_{3}$ ) structures can be approximated as a rectangular QW with infinitely high walls, in which the charge carrier energy is described by Eq. 1.

To confirm the quantum nature of the thicknessdependent oscillations, $\Delta d$ values were calculated theoretically using Eq. 2 and compared with the experimental data. For all films, the oscillation periods calculated using the effective mass approximation and the model of a rectangular quantum well with infinitely high walls and taking into account the $d$-dependence of the Fermi energy and the contribution of all energy subbands located below the Fermi level to the kinetic coefficients were in good agreement with the experimentally observed oscillation periods. This confirms the quantum origin of these oscillations.

For the films prepared from stoichiometric $p-\mathrm{PbSe}$ crystals, oscillatory behavior of the dependences of the kinetic coefficients on $d$ was observed in the thickness range $d<\sim 60 \mathrm{~nm}$. The dominant carrier sign inversion occurring near $d \approx 20 \mathrm{~nm}$ made it possible to investigate QSEs determined by the behavior of both electron and hole gases using the 

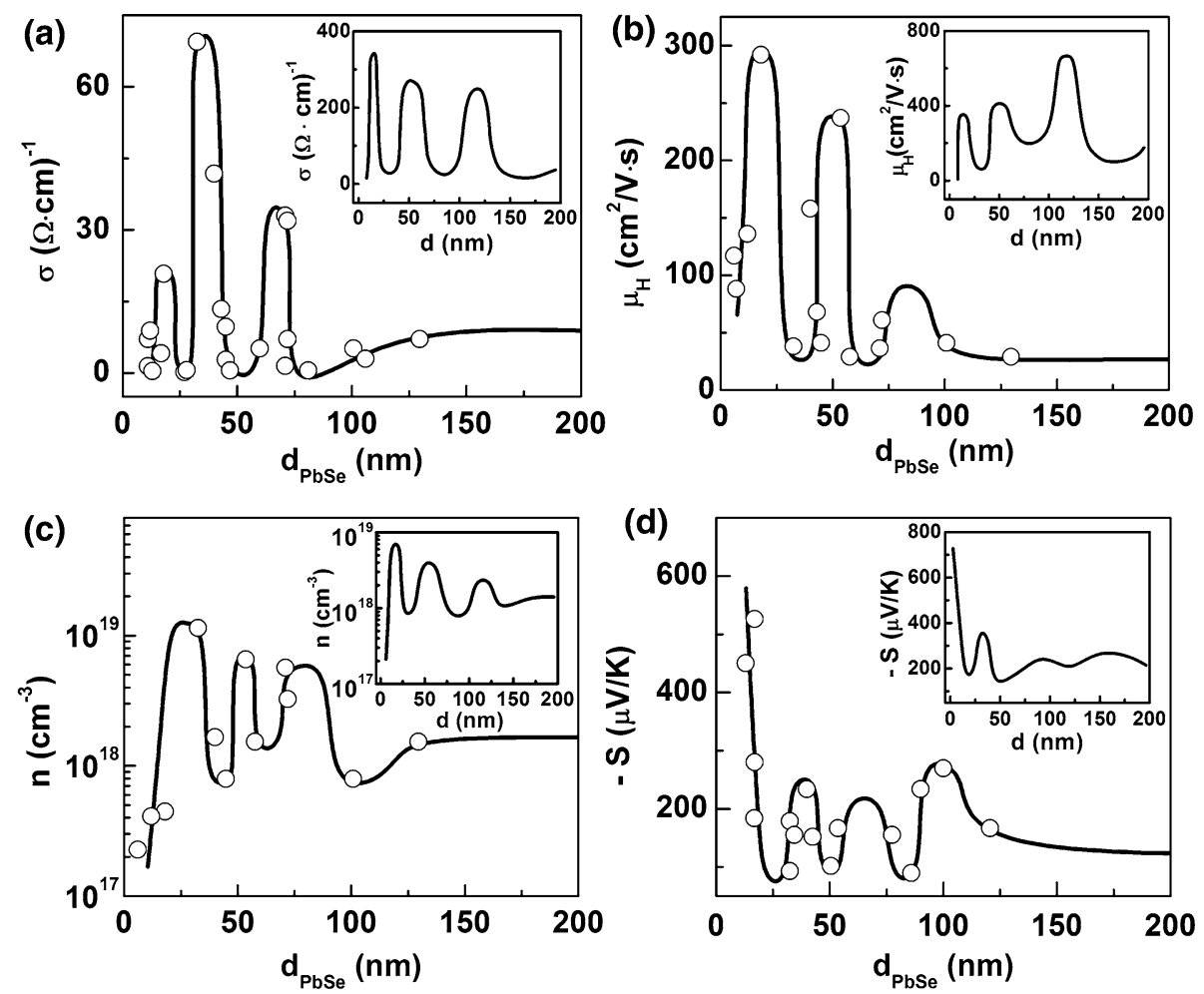

Fig. 4. Dependences of electrical conductivity $\sigma$ (a), electron mobility $\mu_{\mathrm{H}}$ (b), concentration $n$ (c), and the Seebeck coefficient $S$ (d) on total PbSe layers thickness $d$ in PbSe/EuS superlattices. ${ }^{21,22}$ In the insets: the thickness dependences of $\sigma, \mu_{\mathrm{H}}, n$, and $S$ for single PbSe/EuS quantum wells reported in Refs. 15-17.

same specimen. For the films in the $p$-region, the experimentally determined oscillation period $\left(\Delta d_{\text {exp }}=10 \pm 1 \mathrm{~nm}\right)$ was in good agreement with the theoretically calculated value $\left(\Delta d_{\text {calc }}=10 \pm\right.$ $2 \mathrm{~nm}$ ). For the films in the $n$-region, the experimentally measured oscillation period $\left(\Delta d_{\exp }=20 \pm\right.$ $2 \mathrm{~nm}$ ) was also consistent with the result of the theoretical calculations $\left(\Delta d_{\text {calc }}=20 \pm 1 \mathrm{~nm}\right) .{ }^{14}$

For the films grown from nonstoichiometric $n$ PbSe crystals, the experimental and theoretical values of the oscillation period were $\Delta d_{\exp _{5}}=20 \pm$ $2 \mathrm{~nm}$ and $\Delta d_{\text {calc }}=20 \pm 2 \mathrm{~nm}$, respectively. ${ }^{15-17}$

For the $\mathrm{PbSe}\langle\mathrm{Cl}\rangle$ films, oscillatory behavior of the dependences of the kinetic coefficients on $d$ was observed in the thickness range $d<\sim 30 \mathrm{~nm}$ (Fig. 3c). ${ }^{18}$ The theoretical value $\Delta d_{\text {calc }}$ calculated using the model of a rectangular QW with infinitely high walls $(\Delta d=3.0 \pm 0.5 \mathrm{~nm})$ was significantly smaller than the experimental one $\left(\Delta d_{\exp }=7 \pm\right.$ $1 \mathrm{~nm}$ ), however taking into account the $d$-dependence of the Fermi energy ${ }^{27}$ and the contributions to the kinetic coefficients of all energy subbands located below $\varepsilon_{\mathrm{F}}{ }^{28}$ we obtained the value $\Delta d=5.0 \pm 0.5 \mathrm{~nm}$, much closer to the experimental one. ${ }^{18-20}$ For the films prepared using nondoped stoichiometric and nonstoichiometric PbSe crystals as initial material, these factors had practically no effect on the calculated value of $\Delta d$.

It follows from the data obtained that, by investigating the oscillating character of the dependences of kinetic properties on film thickness, one can obtain information about the electron and hole energy spectrum in a $Q W$, determine or verify semiconductor parameters, and compare their values with corresponding values for bulk crystals; For example, having determined the oscillation period, one can verify the effective mass value at given Fermi energy or verify the Fermi energy at given effective mass. By measuring the oscillation period, it is possible to determine the film thickness for which the bottom of the first energy subband crosses the Fermi level and, consequently, the QW energy spectrum. By changing the charge carrier concentration in a film (for example, by doping) and measuring the oscillation period, one can examine the range of applicability of the infinitely deep well model by comparing the oscillation periods for different concentrations.

It is important to note that the dependence of the TE power factor $P$ on the film thickness, which determines to a great extent the efficiency of a TE energy converter, also has oscillatory character (Fig. 2), which should be taken into consideration when developing thin-film materials for use in $\mathrm{TE}$ converters.

\section{Quantum Size Effects in PbSe-Based Superlattices}

It is also interesting to compare the behavior of the thickness dependences of the transport 
properties in single $\mathrm{PbSe} \mathrm{QWs}$ and $\mathrm{PbSe} / \mathrm{EuS}$ SLs grown on (001)KCl substrates.

Figure 4 presents the dependences of the kinetic coefficients on the total thickness $d$ of all $\mathrm{PbSe}$ layers for the $n$-PbSe/EuS SLs. ${ }^{21,22}$ All the dependences have oscillatory character with mean value of the oscillation period $\Delta d=22 \pm 3 \mathrm{~nm}$, in good agreement with the experimentally determined $(\Delta d=20 \pm 5 \mathrm{~nm})$ and theoretically calculated values of $\Delta d$ for single QWs (see the insets in Fig. 4). One can assume that, for both the PbSe/EuS SLs and for the single $\mathrm{PbSe} / \mathrm{EuS} \mathrm{QWs}$, the observed oscillatory behavior of the $d$-dependences of the transport properties can be interpreted in terms of quantum-mechanical concepts associated with gradual filling of lateral subbands with increasing $d$.

Thus, the experimentally determined periods of the thickness-dependent quantum oscillations of the transport properties in the $\mathrm{PbSe} / \mathrm{EuS}$ single QWs and $\mathrm{PbSe} / \mathrm{EuS}$ SLs practically coincide and are consistent with the results of theoretical calculations. The difference between QWs and SLs consists in the fact that, in SLs, one should consider the total thickness of all PbSe layers, which can be changed either by varying the PbSe layer thickness or by varying the number of periods. It follows from this fact that the barrier width is sufficiently small $(10 \mathrm{~nm})$ for realization of tunneling processes. It is generally assumed that, if the potential barrier width is smaller than the de Broglie wavelength $\lambda_{\mathrm{D}}$, then an electron can tunnel through the barrier with a certain probability. Taking into account that the measured oscillation periods for $p$ - and $n$-type $\mathrm{PbSe}$ are $\Delta d=10 \mathrm{~nm}$ and $\Delta d=20 \mathrm{~nm}$, respectively, and that $\Delta d=\lambda_{\mathrm{D}} / 2$, the condition $R<\lambda_{\mathrm{D}}$ is fulfilled. Besides, there are a number of works (see, for example, Refs. 29-31) in which vertical (along the growth direction of the multilayers) electron transport through $\mathrm{PbS} / \mathrm{EuS}$ structures, where a 2-nm- to 10-nm-thick EuS layer played the role of a barrier, was studied. It was shown ${ }^{29-31}$ that current-voltage characteristics had nonlinear character in all cases, indicating electron tunneling from $n$-PbS layers via the spin-dependent EuS ferromagnetic barrier. It was also shown that, with increasing temperature, the barrier height decreased due to a temperature dependence of the band structure parameters of the EuS/PbS heterointerface, which increased the tunneling probability.

It should be noted that, in our SLs, the charge is carried by electrons, which move along the film surface. The experimental fact that the $d$-dependences of kinetic properties for SLs exhibit oscillatory behavior when $d$ is interpreted as the total thickness of all PbSe layers supports the assumption that tunneling through EuS layers takes place. In single PbSe layers with thicknesses corresponding to the thicknesses of separate PbSe layers in the studied PbSe/EuS SLs $(0.5 \mathrm{~nm}$ to $15 \mathrm{~nm})$, QSEs would not be observed because $\Delta d>d$. This is why the conditions for quantization must be applied to the total thickness rather than to the thickness of a separate layer.

In the first theoretical studies of QSEs in thin films, the films were assumed to have perfect surfaces. Later, a number of authors pointed out that a significant role in the manifestation of quantum oscillations is played by surface roughness scattering. It was even mentioned that broadening of energy levels caused by such scattering can subdue oscillations. Considering the high sensitivity of QSEs to surface quality, one can conclude that the clearly observed oscillations in PbSe films provide evidence for high structural perfection of the studied films. Combined with the specular character of the carrier scattering, which is typical of the PbSe surface, favorable conditions for observing QSEs are created. It can also be concluded from the experimental results that, under certain conditions (sufficiently high charge carrier density and sufficiently low $m^{*}$ ), observation of QSEs is quite possible even for epitaxial films grown in island fashion. However, this growth mode complicates determination of the oscillation period $\Delta d$ values when they are small.

The fact that the quantum oscillations of kinetic coefficients are manifested quite distinctly even at room temperature, although it is commonly accepted ${ }^{5-8}$ that any reliable observation of oscillations is possible only at low temperatures, may imply, on the one hand, a rather high degree of structural perfection of the films, and, on the other hand, the possible influence of other (as yet unknown) factors. One can suggest that electron processes accompanying the size quantization may affect the lattice subsystem by stabilizing the structure at critical $d$ values satisfying the quantization conditions. Recent studies on the mechanism of metallic film growth on an insulator ${ }^{32,33}$ support this proposition.

\section{CONCLUSIONS}

This overview and analysis of our experimental data obtained over a number of years from studies of QSEs in thin films of semiconductor PbSe compound, carried out with a view to establishing the effect of various factors (conductivity type, charge carrier concentration, etc.) on the character of QSEs, leads to the following conclusions:

- Studying QSEs in PbSe films is possible only if films are covered with a protective layer.

- The presence of oscillations in the thickness dependences of the kinetic coefficients, which we observed for the first time in $2002^{15}$ in $n$-PbSe films and interpreted as a manifestation of QSEs, has been confirmed by subsequent studies of films prepared from stoichiometric, nonstoichiometric, and doped PbSe crystals with different conductivity types and charge carrier concentrations. In all cases, experimentally determined oscillation periods are in good agree- 
ment with $\Delta d$ values calculated theoretically using the model of a rectangular potential well with infinitely high walls and taking into account the $d$-dependence of the Fermi energy and the contributions to the kinetic coefficients from all energy subbands located below the Fermi level. This consistency between the theoretical and experimental results represents a convincing argument in favor of the interpretation of these data in terms of size quantization.

- The conductivity type in PbSe films can be determined by both the dominant carrier type and charge carrier concentration in the initial crystal, as well as by the film thickness. In the latter case (e.g., thin films grown from stoichiometric $p$-PbSe crystals), by changing $d$, one can study QSEs related to both the electron and hole gas, using the same initial material. If, on changing $d$, inversion of the conductivity sign takes place in the thickness range near the inversion point, thickness-dependent quantum oscillations are accompanied by a periodic change in the conductivity sign. This interesting phenomenon is attributed to high sensitivity of the system to external factors (including changes in $d$ ) near the inversion point.

- Quantum oscillations in the thickness dependences of transport properties are also observed for $n$-PbSe/EuS superlattices, and the oscillation period coincides (within the limits of error) with $\Delta d$ for $n$-PbSe single films with thickness equal to the total thickness of all PbSe layers in the superlattice. This fact is attributed to electron tunneling through EuS barriers.

- At sufficiently high carrier density and sufficiently low $m^{*}$, observation of QSEs in the transport properties is quite possible for epitaxial films grown not only layer-by-layer but even in island fashion.

- The thickness-dependent oscillations in the transport properties distinctly manifest themselves at room temperature. This fact, on the one hand, may indicate rather high structural quality of the films, and, on the other, may imply the influence of other factors.

- Studying the $d$-dependences of transport properties is a relatively simple and reliable method for investigating the energy spectrum of electrons in a QW, especially if combined with other methods (optical, tunneling, etc.).

- The observed oscillatory character of the thickness dependences of the transport properties should be taken into account when designing devices based on thin-film structures, for which quantum-mechanical effects are relevant.

\section{ACKNOWLEDGEMENT}

This work was supported by the Ukrainian Ministry of Education and Science (Project No. M3925).

\section{REFERENCES}

1. J.H. Davies, The Physics of Low-Dimensional Semiconductors. An Introduction (New York: Cambridge University Press, 1998).

2. M.J. Kelley, Low-Dimensional Semiconductors: Materials, Physics, Technology, Devices (Oxford: Clarendon, 1995).

3. I.M. Lifshits and A.M. Kosevich, Izv. AN USSR. Ser. Fiz. 19, 395 (1955).

4. Y.I. Ravich, B.A. Efimova, and I.A. Smirnov, Semiconducting Lead Chalcogenides (New York: Plenum, 1970).

5. B.A. Tavger and V.Ya. Demihovskii, Usp. Fiz. Nauk. 96, 61 (1968).

6. V.N. Ogrin, M.I. Lutskii, and O. Elinson, Pis'ma $v$ ZhETF 3, 114 (1966).

7. O.N. Filatov and I.A. Karpovich, Pis'ma $v$ ZhETF 10, 142 (1969).

8. L. Zdanowicz, W. Zdanowicz, and G. Pocztowski, Thin Solid Films 28, 345 (1975). doi:10.1016/0040-6090(75) 90125-X.

9. D.M. Rowe, CRC Handbook of Thermoelectrics (Boca Raton: CRC Press, 1995).

10. L.D. Hicks and M.S. Dresselhaus, Phys. Rev. B 47, 12727 (1993). doi:10.1103/PhysRevB.47.12727.

11. T.C. Harman, P.J. Taylor, D.L. Spears, and M.P. Walsh, in Proceedings of 18th International Conference on Thermoelectrics, Baltimore, USA, 1999, p. 280 . doi: 10.1109/ICT.1999.843386.

12. E.I. Rogacheva, T.V. Tavrina, S.N. Grigorov, O.N. Nashchekina, V.V. Volobuev, A.G. Fedorov, K.A. Nasedkin, and M.S. Dresselhaus, J. Electron. Mater. 31, 298 (2002). doi:10.1007/s11664-002-0147-y.

13. E.I. Rogacheva, T.V. Tavrina, O.N. Nashchekina, S.N. Grigorov, AYu Sipatov, V.V. Volobuev, M.S. Dresselhaus, and G. Dresselhaus, Phys. E 17, 310 (2003). doi: 10.1016/S1386-9477(02)00819-6.

14. E.I. Rogacheva, O.N. Nashchekina, S.I. Ol'khovskaya, and M.S. Dresselhaus, J. Thermoelectr. 4, 25 (2012).

15. E.I. Rogacheva, T.V. Tavrina, O.N. Nashchekina, S.N. Grigorov, K.A. Nasedkin, M.S. Dresselhaus, and S.B. Cronin, Appl. Phys. Lett. 80, 2690 (2002). doi: 10.1063/1.1469677.

16. E.I. Rogacheva, T.V. Tavrina, O.N. Nashchekina, S.N. Grigorov, K.A. Nasedkin, M.S. Dresselhaus, and S.B. Cronin, Virtual J. Nanoscale Sci. Technol. 5, 16 (2002).

17. E.I. Rogacheva, O.N. Nashchekina, T.V. Tavrina, M.A. Us, M.S. Dresselhaus, S.B. Cronin, and O. Rabin, Phys. E 17, 313 (2003). doi:10.1016/S1386-9477(02)00820-2.

18. S.I. Menshikova, E.I. Rogacheva, AYu Sipatov, S.I. Krivonogov, and P.V. Mateychenko, J. Thermoelectr. 2, 22 (2015).

19. S.I. Ol'khovskaya, E.I. Rogacheva, and AYu Sipatov, Metallofiz. Noveishie Tekhnol. 33, 213 (2011).

20. S. Ol'khovskaya and O. Rogachova, Visnyk Lviv Univ. Ser. Phys. 47, 88 (2012).

21. E.I. Rogacheva, O.N. Nashchekina, AYu Sipatov, A.G. Fedorov, S.N. Grigorov, T.V. Tavrina, and M.S. Dresselhaus, Phys. Status Solidi C 6, 1149 (2009). doi: 10.1002/pssc.200881229.

22. E.I. Rogacheva, O.N. Nashchekina, S.I. Ol'khovskaya, A.Y. Sipatov, and M.S. Dresselhaus, in Proceedings of 31st International Conference on the Physics of Semiconductors, ICPS 2012, Zurich, Switzerland, 1566, 2013, pp. 207-208. doi:10.1063/1.4848358.

23. M.H. Brodsky and J.N. Zemel, Phys. Rev. 155, 780 (1967). doi:10.1103/PhysRev.155.780.

24. R.F. Egerton and C. Juhasz, Thin Solid Films 4, 239 (1969). doi:10.1016/0040-6090(69)90070-4.

25. G.F. McLane and J.N. Zemel, Thin Solid Films 7, 229 (1971). doi:10.1016/0040-6090(71)90070-8.

26. E. Rogacheva, O. Vodorez, O. Nashchekina, A. Sipatov, A. Fedorov, and S. Olkhovskaya, J. Electron. Mater. 39, 2085 (2010).

27. V.D. Dymnikov, Phys. Solid State 53, 901 (2011). 
28. M.P. Singh and C.M. Bhandari, Solid State Commun. 133, 29 (2005).

29. L. Kowalczyk, and J. Sadowski, et al., Acta Phys. Pol. A 94, 397 (1998).

30. S. Wroteka, et al., Acta Phys. Pol. A 103, 629 (2003).
31. S. Wroteka, A. Morawskia, and Z. Tkaczyk, et al., Acta Phys. Pol. A 105, 815 (2004).

32. Z.Y. Zhang, Q. Niu, and C.-K. Shih, Phys. Rev. Lett. 80, 5381 (1998).

33. J.-H. Cho, K.S. Kim, C.T. Chan, and Z. Zhang, Phys. Rev. B 63, $113408(2001)$ 